\title{
Iodination of Single-Walled Carbon Nanotubes
}

Karl S. Coleman, ${ }^{* 1}$ Amit K. Chakraborty, ${ }^{1}$ Sam R. Bailey, ${ }^{2}$ Jeremy Sloan, ${ }^{3}$ Morgan Alexander ${ }^{4}$

${ }^{1}$ Department of Chemistry, University of Durham, South Road, Durham, UK, DH1 3LE.

${ }^{2}$ Department of Chemistry, University of Oxford, South Parks Road, Oxford, UK, OX1 3QR.

${ }^{3}$ School of Electronics \& Physical Sciences, University of Surrey, Guildford, UK, GU2 7XH.

${ }^{4}$ School of Pharmacy, University of Nottingham, University Park, UK, NG7 2RD.

Email: k.s.coleman@durham.ac.uk

\section{Supplementary Information}
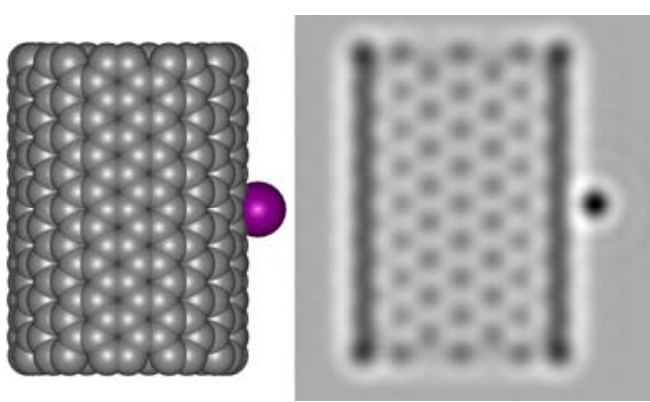

Figure S1. TEM contrast image simulation of an individual iodine atom bound to the surface of a SWNT.

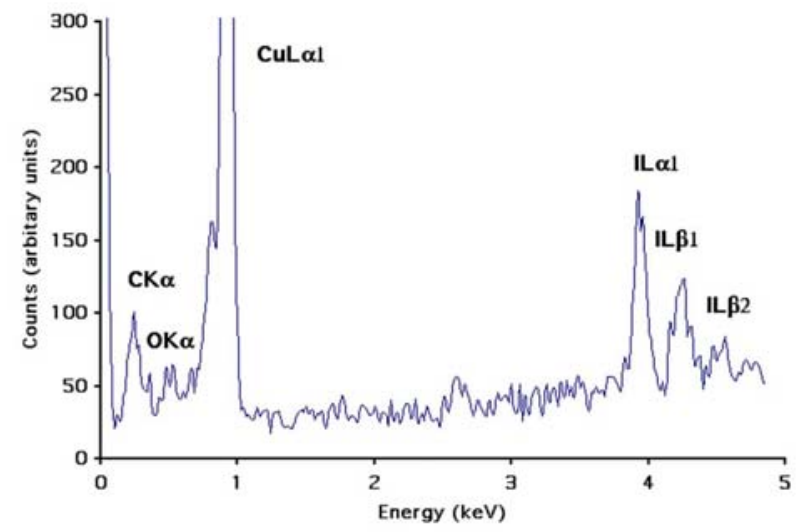

Figure S2. Wide area EDX spectrum of SWNT-I 


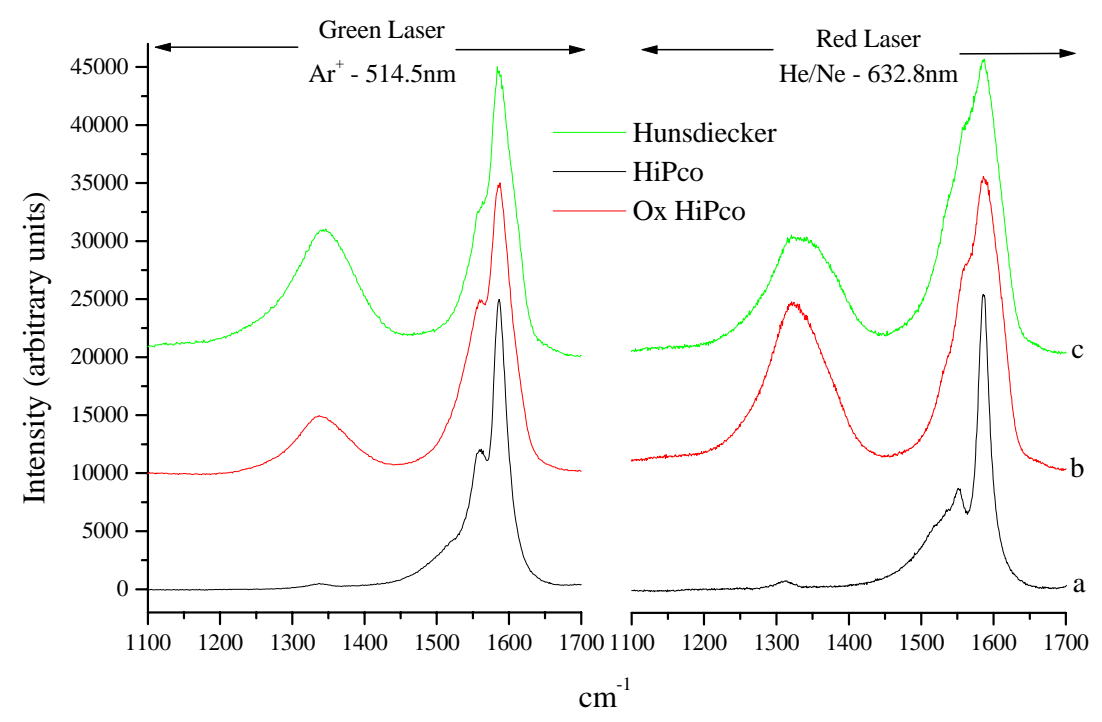

Figure S3. Comparison of the $1100-1700 \mathrm{~cm}^{-1}$ region of the Raman spectrum recorded using an $\mathrm{Ar}^{+}$laser (514.5nm) and a He/Ne laser (632.8nm). a) HiPco SWNT starting material, b) Oxidised HiPco SWNT material (SWNT-COOH), c) Hunsdiecker reaction product (SWNT-I). 